\title{
Multiple resources: The concepts of task difficulty and response requirements
}

\author{
FELICIA C. GOLDSTEIN \\ University of Texas Medical Branch, Galveston, Texas \\ and \\ HOWARD A. ROLLINS, JR. \\ Emory University, Atlanta, Georgia
}

\begin{abstract}
The variables of task difficulty and response requirements were examined within the multiple resources framework. The performance of 54 undergraduates was monitored on a visual target detection task as the difficulty of auditory shadowing was manipulated. Response competition was examined by varying the time at which subjects responded to the visual target. In line with multiple resources theory, there were no significant differences in target detection as a function of different demands of auditory shadowing, and there was no evidence for switching between auditory and visual inputs. Subjects were devoting their effort toward shadowing as measured by control trials of shadowing alone. Although evidence for response competition was not obtained in the hypothesized direction, the subjects who responded to visual targets while continuing to shadow performed better than other subjects. Alternative theories and the notion that effort may refer to the range of available processing strategies are discussed.
\end{abstract}

The present investigation tested a prediction of the multiple resources framework that concurrent processing of two inputs should be possible to the extent that they differ in their resource demands. Navon and Gopher (1979) suggested that the attentional system comprises several channels, each having its own capacity. Performance depends upon the type of resources demanded by a task as well as the availability and efficiency of each type of resource (Friedman, Polson, Dafoe, \& Gaskill, 1982). Two recent studies have tested the multiple resources approach to attention. Friedman et al. (1982) presented tasks which overlapped completely, partially, or not at all in their resource demands; performance was poorest when a memory task (utilizing left hemisphere resources) was combined with a detection task presented to the right visual field (left hemisphere resources) versus the left visual field (right hemisphere resources). Gopher, Brickner, and Navon (1982) manipulated priority and difficulty (varied along cognitive or motor demands) for subjects performing pursuit tracking and letter-typing tasks; priorities were affected only when the motor demands of typing were combined with the tracking task, presumably because they competed for common resources.

The present experiment afforded a further test of the multiple resources prediction mentioned above. First,

This study is based upon a thesis submitted to Emory University in partial fulfillment of the requirements for the Doctor of Philosophy degree. The authors thank Larry Barsalou for his valuable input and Daniel Duvarney for programming the computer. Address for reprints: F. C. Goldstein, Department of Neurology, University of Texas Medical Branch, Galveston, TX 77550. rather than utilizing similar types of inputs (e.g., two visual inputs), we selected tasks which differed in their processing requirements. Subjects performed relatively difficult perceptual-memory activities: shadowing of digits and nonverbal visual target detection. According to multiple resources, target detection requires a different set of processors (visual) than does shadowing (speech), and concurrent processing should be possible. Second, the experiment required simultaneous rather than serial processing (Friedman et al., 1982). Simultaneous presentation should not affect information processing to the extent that resources are independent. Third, the study permitted an ongoing assessment of whether allocation strategies of subjects were changing throughout the experiment. The methodology tested whether a switching of attention between shadowing and target detection occurred by examining whether errors in shadowing occurred at the moment a target was detected.

The first variable, resource demand, was manipulated by increasing the difficulty of shadowing (Johnston \& Heinz, 1978, 1979). Errors in detecting visual targets were compared during a control condition of no shadowing; a single-message condition in which one speech input to both ears was shadowed; and a binaural condition in which two speech inputs to both ears were received in different sexed voices, and subjects shadowed one of these messages. The multiple resources position predicts no significant decline in detection to the extent that the tasks do not share resources.

The second variable examined was the influence of different response requirements. Several researchers (Duncan, 1980a, 1980b; McLeod, 1978) have suggested 
that the measurement of capacity within the dual-task paradigm may be confounded by the fact that studies have typically required two ongoing responses. In the current investigation, the groups differed according to whether they responded to targets immediately with continued shadowing or without continued shadowing or at the end of the trial. Relative to subjects during control trials, subjects who responded to a target while also shadowing were expected to make significantly more errors. The influences of differences in memory requirements among the groups (remembering to stop shadowing or remembering to press the key at the end of a trial) were considered to be slight, and, in fact, no subject reported difficulty in remembering how to respond.

\section{METHOD}

\section{Subjects}

The subjects were 54 (15 male, 39 female) right-handed undergraduates enrolled in introductory psychology classes at Emory University. Eighteen subjects served in each time-of-response condition.

\section{Visual and Auditory Stimuli}

Visual targets were generated with Attneave and Arnoult's (1956) Method 1 for the construction of random nonsense shapes. Distractors were then created by perturbing the coordinates of the target points in terms of a user-specified number and distance on an Apple II+ computer (Cooper \& Podgorny, 1976). There were 96 unique six-sided targets, each having a set of 12 unique distractors. On average, the figures subtended vertical and horizontal visual angles of $3^{\circ}$ each. Three sets of stimuli were constructed and counterbalanced across the type of shadowing manipulation. ${ }^{1}$ Twelve figures were presented on a trial via the computer screen at the rate of one figure every $1.56 \mathrm{sec}$ for $200 \mathrm{msec}$ each. On trials in which a target appeared (16 of 32), the target's position was randomly determined with the restriction that it not occur as one of the first two or last two figures.

Auditory stimuli were recorded via Supertalker, an Apple II+ peripheral which digitizes and stores speech. On single-message trials, seven digits ( 1 to 8 , excluding 7 ) recorded in a female voice were presented. On binaural trials, the stimuli were recorded by having a male and female simultaneously repeat two different digits, synchronized as closely as possible to a clicking tone (Johnston \& Heinz, 1978). All possible combinations of digits were recorded (except same digits), making up a pool of 42 pairs. A digit or digit pair was presented every $780 \mathrm{msec}$, and every other digit coincided with the appearance of a visual figure. Shadowing errors were scored in terms of omissions, insertions, incorrect names, stumbles, and mispronunciations as well as intrusions of the male digits in the binaural condition. Inter-rater reliability was high (.99 for the single message and .97 for the binaural conditions).

\section{Procedure}

Subjects were tested individually. In the no-shadowing condition (32 detection trials), the subject was told that a target would come on the screen (actual time $=7.5 \mathrm{sec}$ ) followed by a rapidly presented series of figures. The subject was instructed to press a response button if the target was detected and was informed that there would be trials in which the target would not appear at all. The target appeared once on a random $50 \%$ of the trials.

Subjects in the stop-shadowing and respond-while-shadowing conditions received these instructions. Subjects in the third response condition were told to press the key at the trial's completion if they saw the target. In the single-message condition (32 detection trials, 16 shadowingonly trials), the subject was told to simultaneously repeat out loud digits spoken by a female over earphones. The subject was reminded that a target would not always appear and was instructed to devote as much energy as possible to shadowing. Subjects were also informed that on some trials shadowing only would be required. Three levels of response instructions were given (press the key while continuing to shadow; stop shadowing, press the key, and wait for the next trial; press the key at the end of the trial). The same instructions were given for the binaural condition ( 32 detection trials, 16 shadowing-only trials), except that subjects were required to shadow digits spoken by the female. Each condition was run in blocked fashion and counterbalanced using a Latin square design (Myers, 1979).

\section{RESULTS}

\section{Evaluation of Target Performance}

The proportion of hits (16 possible) and false alarms (16 possible) was used to determine $d^{\prime}$ and beta values for every subject (Freeman, 1964). A 3 (type of shadowing condition) $\times 3$ (time of response) repeated measures ANOVA on $d^{\prime 2}$ revealed a significant main effect of time of response $[F(2,51)=4.91, p<.01]$ but no main effect of type of shadowing and no interaction. Table 1 shows the $d^{\prime}$ values. A post hoc Newman-Keuls analysis indicated significantly smaller values and, hence, less sensitivity $(p<.05)$ for subjects who responded after the trial (mean $=1.43, \mathrm{SD}=.64$ ) than for subjects who responded while shadowing (mean $=2.01, \mathrm{SD}=.84$ ). However, $d^{\prime}$ for the stop-shadowing group (mean $=1.64$, $\mathrm{SD}=.72$ ) was not significantly different from the other conditions. A 3 (type of shadowing condition) $\times 3$ (time of response) repeated measures ANOVA on beta values (Table 1) indicated no significant differences in beta for type of shadowing $[F(2,102)=1.75, p>.05]$, for time of response $[F(2,51)=2.71, p>.05]$, or for the interaction $[F(4,102)=.96, p>.05]$.

In these previous analyses, hit rate was determined by noting whether or not a keypress was made on trials when a target appeared in order to compare the performance of subjects responding during or after the trial. However, subjects responding during a trial may have keypressed to

Table 1

Mean $d^{\prime}$ and Beta Values as a Function of Type of Shadowing Condition and Time of Response

\begin{tabular}{lcccc}
\hline \multirow{2}{*}{$\begin{array}{c}\text { Time of } \\
\text { Response }\end{array}$} & No Shadowing & Single Message & Binaural & Overall Mean \\
\cline { 2 - 5 } & $1.56(1.52)$ & $1.66(3.26)$ & $1.69(1.70)$ & $1.64(2.16)$ \\
Stop Shadowing & $2.07(4.30)$ & $2.14(4.05)$ & $1.81(2.82)$ & $2.01(3.72)$ \\
$\begin{array}{l}\text { Respond While } \\
\text { Shadowing }\end{array}$ & $1.70(1.89)$ & $1.29(1.80)$ & $1.32(1.61)$ & $1.43(1.76)$ \\
After Trial & $1.78(2.57)$ & $1.70(3.04)$ & $1.60(2.04)$ & \\
Overall Mean & & & & \\
\hline
\end{tabular}

Note-beta values are in parentheses. 
a nontarget figure, but the response was recorded as a hit. Obviously, hit rate could be spuriously inflated, and true changes in performance would be masked. "True" target responses are available for the groups responding during the trial by noting when a keypress occurred. A window of $1.56 \mathrm{sec}$ (time between target offset to a new figure) was used to determine whether a response was made to a target, and $d^{\prime}$ and beta were then recalculated.

A 3 (type of shadowing condition) $\times 2$ (time of response) repeated measures ANOVA on "true" $d$ ' values indicated a significant main effect of time of response $[F(1,34)=4.20, p<.05]$. Subjects who responded while shadowing were more sensitive to targets (mean = $1.76, S D=.94$ ) than were subjects who stopped shadowing before responding (mean $=1.28, S D=.79$ ). However, as in the previous analyses, there was neither a main effect of type of shadowing nor an interaction. A 3 (type of shadowing condition) $\times 2$ (time of response) $\times 3$ (order of conditions) repeated measures ANOVA on "true" beta values indicated a significant main effect of time of response $[F(1,30)=4.50, p<.05]$. Overall, the respond-while-shadowing group (mean $=3.99$, $S D=3.81$ ) was more cautious in responding than was the stop-shadowing group (mean $=2.43, S D=1.96$ ). In addition, when the order of conditions was binaural, single message, and no shadowing, the respond-whileshadowing group was significantly $(p<.05)$ more cautious (mean $=6.04$ ) than was the stop-shadowing group $($ mean $=2.10)$.

Another possibility is that the experiment may not have been sensitive enough to real differences in $d^{\prime}$ over various shadowing conditions. A frequency count was performed for the respond-while-shadowing and stopshadowing groups on $d^{\prime}$ for the most extreme ends: no shadowing versus binaural shadowing. Twelve subjects in the stop-shadowing group had higher $d^{\prime}$ values and 6 subjects had lower $d^{\prime}$ values in the binaural as opposed to the no-shadowing condition. For the respond-duringtrial group, 8 subjects had higher $d^{\prime}$ scores and 10 had lower $d^{\prime}$ scores for the same comparison. A chi-square test of independence revealed no significant differences between the number of subjects with higher or lower $d^{\prime}$ values (stop shadowing: $\chi^{2}(1)=1.00, p>.05$; respond during trial: $\left.\chi^{2}(1)=.22, p>.05\right)$.

Finally, reaction time data were available for subjects who responded while shadowing or who stopped shadowing and then responded. Capacity theorists have utilized reaction time as a sensitive indicator of interference. There was no significant main effect of latency for type of shadowing (no shadowing, mean $=.887$; single message, mean $=.861$; binaural shadowing, mean $=.895$ ) or time of response (respond while shadowing, mean $=.870$; stop shadowing, mean $=.893$ ).

\section{Evaluation of Shadowing Performance}

A 2 (type of shadowing) $\times 3$ (time of response) $\times 3$ (order of conditions) repeated measures ANOVA was performed on the proportion of shadowing errors. There was a significant main effect of type of shadowing $[F(1,45)$ $=96.90, p<.01]$. Subjects in all response groups made more errors when repeating the binaural message (mean $=.13, S D=.71)$ than the single message $($ mean $=.02$, $S D=.24)$, indicating that difficulty of shadowing was indeed manipulated.

A further analysis evaluated whether subjects were truly devoting all of their attention to the shadowing task. Since the errors made in the single-message condition were extremely low, it was felt that a truer evaluation would occur for the binaural condition. A 2 (type of shadowing) $\times 3$ (time of response) $\times 3$ (order of conditions) repeated measures ANOVA revealed no significant main effect of shadowing errors in a comparison of auditory trials alone (mean $=.12, S D=.07$ ) and auditory plus visual trials $($ mean $=.13, S D=.07){ }^{3}$

Finally, it was possible to determine the exact digit the subject repeated at the exact moment the target appeared on the screen for the stop-shadowing and respond-whileshadowing groups. To the extent that a subject was switching attention, one would expect to find a positive correlation between target detection and shadowing errors (as a target is detected, a shadowing error should be made as well). For every subject, the number of true hits and the number of shadowing errors made at the moment the target appeared were determined. The Pearson product moment correlation between true hits and shadowing errors was . 15 for subjects who stopped shadowing and .09 for those who responded while shadowing, both not significantly different from zero.

\section{DISCUSSION}

The data are supportive of a multiple resources position that parallel processing of two inputs is possible despite an increase in the capacity they require. The concepts of task difficulty and effort (Kahneman, 1973) are less predictive of performance. These findings, although supportive of the null hypothesis, are intuitively surprising when one considers how difficult the tasks were. First, the visual task had a memory component which required a subject to remember the initial target figure. In addition, the task was difficult as judged by the relatively high number of missed targets in the control condition of no shadowing. Finally, even when looking at the pattern of $d^{\prime}$ values for individual subjects in the no-shadowing versus the binaural-shadowing conditions, $d^{\prime}$ was not consistently lower in the latter condition.

A potential alternative theoretical basis for the lack of interference is the capacity model which incorporates the concepts of data-limited and resource-limited tasks (Norman \& Bobrow, 1975). A data-limited task is one in which the further allocation of processing resources has no effect on performance. A resource-limited task, on the other hand, is one in which performance improves as the resources allocated to the task are increased. Perhaps the visual target detection task is data-limited such that slower presentation rates of the figures or prolonged study time for the initial target figure would not have improved performance. In such a case, the increased resource demands placed on subjects during shadowing would have no impact on detection rates for the datalimited visual task. However, there is some evidence that the visual task is resource-limited. Pilot subjects were run on the detection task in order to establish a $75 \%$ hit rate. More targets were correctly detected as the duration of each target increased. If one assumes that a longer stimulus duration allows more time for resources to be allocated, then these pilot data suggest that the task is resource-limited.

An analysis of "true" target performance showed that the stopshadowing subjects were less sensitive to the appearance of targets and 
less cautious in making a response than were subjects who responded while shadowing. Thus, stopping shadowing may have been disruptive. Researchers (Rollins \& Hendricks, 1980; Treisman \& Riley, 1969) have traditionally employed the stop-shadowing condition with the idea that it eliminates a response. The requirement to stop shadowing may have placed an additional decisional load on subjects or had its disruptive influence by making subjects inhibit an ongoing response. The present finding supports the views and results of other researchers (Duncan, 1980a, 1980b; McLeod, 1977) that response requirements may show an inconsistent pattern when an identical task is utilized for two groups and should caution those using dual-task methodology to consider the possibility of response interference in interpreting their results.

It seems unlikely that the number of trials was extensive enough to enable one task to become either so skilled (Neisser, 1976) or so automatic (Schneider \& Fisk, 1982) that parallel processing was possible. However, an untested hypothesis and one which has recently been suggested (Friedman \& Polson, 1981) is that "practice" may actually refer to a change in processing strategies in order to permit the handling of simultaneous inputs. For example, some subjects may have initially tried to recognize figures by labeling the target, but when shadowing, this strategy was harmful to performance and they switched their strategy. Such a position carries with it the idea that difficulty of a task may actually refer to the range of processing strategies left open to an individual when confronted with an input. The fewer the options, the more difficult the task may become when combined with another task which also requires a similar strategy.

\section{REFERENCES}

Attneave, F., \& Arnoult, M. (1956). The quantitative study of shape and pattern perception. Psychological Bulletin, 53, 452-471.

COOPER, L., \& PODGORNy, P. (1976). Mental transformations and visual comparison processes: Effects of complexity and similarity. Journal of Experimental Psychology: Human Perception \& Performance, 2, 503-514.

Duncan, J. (1980a). The demonstration of capacity limitation. Cognitive Psychology, 12, 75-96.

DunCan, J. (1980b). The locus of interference in the perception of simultaneous stimuli. Psychological Review, 87, 272-300.

Freeman, P. R. (1964). Table of $d^{\prime \prime}$ and beta. Cambridge, England: Medical Research Council.

Friedman, A., \& Polson, M. C. (1981). Hemispheres as independent resource systems: Limited-capacity processing and cerebral specialization. Journal of Experimental Psychology: Human Perception \& Performance, 7, 1031-1058.

Friedman, A., Polson, M., Dafoe, C., \& Gaskill, S. (1982). Dividing attention within and between hemispheres: Testing a multiple resources approach to limited-capacity information processing. Journal of $E x$ perimental Psychology: Human Perception \& Performance, 8, 625-650.

GOPHER, D., BrickNer, M., \& NAvoN, D. (1982). Different difficulty manipulations interact differently with task emphasis: Evidence for multiple resources. Journal of Experimental Psychology: Human Perception \& Performance, 8, 146-157.
Johnston, W. A., \& Heinz, S. P. (1978). Flexibility and capacity demands of attention. Journal of Experimental Psychology: General, 107, 420-435.

JoHnston, W. A., \& HeINZ, S. P. (1979). Depth of nontarget processing in an attention task. Journal of Experimental Psychology: Human Perception \& Performance, 5, 168-175.

Kahneman, D. (1973). Attention and effort. Englewood Cliffs, NJ: Prentice-Hall.

McLEOD, P. (1977). A dual task response modality effect: Support for multiprocessor models of attention. Quarterly Journal of Experimental Psychology, 29, 651-667.

McLeod, P. (1978). Does probe RT measure central processing demand? Quarterly Journal of Experimental Psychology, 30, 83-89.

Myers, J. L. (1979). Fundamentals of experimental design. Boston: Allyn \& Bacon.

NAvoN, D., \& Gopher, D. (1979). On the economy of the humaninformation processing system. Psychological Review, 86, 214-255.

NeISSER, U. (1976). Cognition and reality. San Francisco: Freeman.

Norman, D. A., \& Bobrow, D. G. (1975). On data-limited and resource-limited processes. Cognitive Psychology, 7, 44-64.

Roluins, H. A., \& Hendricks, R. (1980). Processing of words presented simultaneously to ear and eye. Journal of Experimental Psychology: Human Perception \& Performance, 6, 99-109.

SCHNEIDER, W., \& FisK, A. D. (1982). Concurrent automatic and controlled visual search: Can processing occur without resource cost? Journal of Experimental Psychology: Learning, Memory, \& Cognition, 8, 261-278.

Treisman, A. M., \& Riley, J. G. (1969). Is selective attention selective perception or selective response? A further test. Journal of $E x$ perimental Psychology, 79, 27-34.

\section{NOTES}

1. Preliminary ANOVAs were performed to determine whether the visual sets differed in difficulty. There was no statistically significant main effect of set difficulty for any dependent measure.

2. Preliminary repeated measures ANOVAs were run with the main factors of interest, type of shadowing, and time of response, and the counterbalanced variable, order of conditions. Order of conditions was significant for measures of "true" beta and shadowing performance and therefore was retained in these analyses.

3. Two order effects occurred which do not alter the findings that binaural shadowing was more difficult than single-message shadowing and that the proportion of errors made on binaural-only trials was comparable to those on binaural-plus-visual trials. When the binaural condition occurred last, subjects who responded while shadowing tended to make fewer shadowing errors in general than did subjects who responded after the trial. The proportion of errors made by subjects who stopped shadowing, however, was not significantly different from errors made by the other two groups of subjects.

(Manuscript received for publication December 18, 1985.) 